
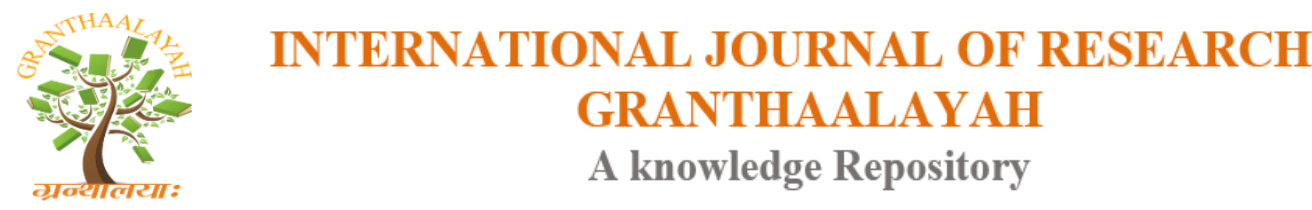

Science

\title{
CATALASE INTRINSIC EMISSIONS OF ELECTROMAGNETIC FIELDS AS PROBABLE CAUSE IN CANCEROGENESIS FROM CONSUMPTION OF RED AND PROCESSED MEAT
}

\author{
Abraham A. Embi *1 \\ *1 13442 SW 102 Lane, Miami, Florida, USA 33186
}

\begin{abstract}
In 2015, reports by The International Agency for Research on Cancer warned of a high incidence of cancer due to processed meats intake. At present, the etiologic factor(s) behind this cancer genesis (is)(are) unknown. The purpose of this manuscript is to introduce a hypothesis linking the protein enzyme catalase (CAT) added to processed meats during manufacturing as a probable cause for the higher cancer incidence. CAT is added during the processed food manufacturing for esthetic reasons, to avoid the "bleaching effect" by hydrogen peroxide. Supporting the hypothesis are prior experiments linking electromagnetic fields (EMFs) emissions during cell respiration, as causing DNA damage and triggering cancer. In that research (CAT) was briefly reported to emit electromagnetic fields (EMFs). This manuscript reintroduces and emphasizes the ubiquitous enzyme catalase as possible cause in cancer genesis reported from processed meat consumption. Additional experiments are presented where a Prussian Blue Solution (PBS) was mixed with two parts of fine iron particles 2000 nanometers $(\mathrm{nm})$ in diameter. Control experiments had human hairs mounted on a slide and then exposed to the liquid PBS. A slide assembly was constructed that EMFs emissions by catalase proper disrupting the metabolic process of the human hair follicle. CAT is shown to be an independent emitter of EMFs and therefore hypothesized as cancerogenic to the human body.
\end{abstract}

Keywords: Catalase; Electromagnetic Forces; Processed Meats; Tissue Metabolism; Cancer Genesis.

\section{List of Abbreviations:}

CAT $=$ Protein enzyme catalase.

Diamagnetic $=$ Property of substance that repels magnetic fields.

EMFs = Electromagnetic Fields.

$\mathrm{Fe} 2 \quad=$ Iron molecule in diamagnetic Prussian Blue Stain.

$4 \mathrm{~K}=$ Two parts of fine iron particles 2000 nanometers in diameter.

$\mathrm{H} 2 \mathrm{O} 2=$ Hydrogen Peroxide.

Nanometer $=$ Unit of measurement equals to 1 millionths of a meter.

PBS = Prussian Blue Stain.

PBS Fe $24 \mathrm{~K}=$ Working solution containing iron particles used to detect EMFs. 
Cite This Article: Abraham A. Embi. (2018). "CATALASE INTRINSIC EMISSIONS OF ELECTROMAGNETIC FIELDS AS PROBABLE CAUSE IN CANCEROGENESIS FROM CONSUMPTION OF RED AND PROCESSED MEAT." International Journal of Research Granthaalayah, 6(8), 33-40. https://doi.org/10.29121/granthaalayah.v6.i8.2018.1259.

\section{Introduction}

In 2015, reports by The International Agency for Research on Cancer warned of a high incidence of cancer due to processed meats intake (1). At present, the etiologic factors behind processed foods and the cancer genesis are unknown. The purpose of this manuscript is to introduce a hypothesis linking the protein enzyme catalase (CAT) that is added to processed meats during manufacturing as a probable cause. CAT is added during the processed food manufacturing for esthetic reasons, to avoid the "bleaching effect" by hydrogen peroxide. Supporting the hypothesis are prior experiments linking electromagnetic fields (EMFs) emissions during cell respiration, as causing DNA damage and triggering cancer genesis $(2,3)$. In those papers (CAT) was briefly reported to emit electromagnetic fields (EMFs). This manuscript reintroduces and emphasizes the ubiquitous enzyme catalase as possible cause in cancer genesis reported in processed foods. Prussian Blue Solution (PBS) was mixed with two parts of fine iron particles 2000 nanometers (nm) in diameter (4). Control experiments had human hairs mounted on a slide and exposed to the liquid PBS (5). A slide assembly was constructed that confirmed EMFs emissions by catalase proper disrupting the metabolic process of the human hair follicle. CAT then, as an independent emitter of EMFs could disrupt the homeostasis of the eukaryotic cell. In the USA catalase used in processed foods are regulated, not inspected.

\subsection{The Hypothesis}

The Electromagnetic Fields emitted by the protein enzyme catalase ubiquitously present in processed meat products are identified as probable factor in cancer genesis.

\subsection{Experiments supporting the Hypothesis}

In this manuscript we expand the research on the disruptive effect of the diamagnetic protein enzyme catalase (CAT) EMFs penetrating a glass barrier on a biological tissue sample. The discovery of in vitro EMFs emitted during CAT dissociation of $\mathrm{H}_{2} \mathrm{O}_{2}$; led to a paper linking Reactive Oxygen Species reactions (EMFs) and cancer. In this manuscript the role of CAT proper as an emitter of EMFs is reintroduced, and hypothesized to be a factor in cancer genesis when introduced in the human digestive system via processed food.

The human hair follicle has been described as a mini-organ with complex cellular divisions, its own circulatory system and metabolic activity (6). The metabolic process entails energy (electron movement) that according to Faraday' law and the Hall effect (7) induces electromagnetic fields (EMFs). The human hair follicle biomagnetism was introduced by D. Cohen in1980 (8). Additional experiments in 2016 demonstrated the hair follicle EMFs penetrating vertically through a $1 \mathrm{~mm}$ glass barrier [9]. Also shown, were metabolic disruptions of fronted hair follicles when imbedded in a diamagnetic (repulsive) solution (10). As aforementioned, EMFs emitted during cell respiration were proposed to be an additional factor in cancer genesis as cited in (3). 


\section{Materials and Methods}

Prussian Blue Stain solution was prepared by using two parts of Potassium Ferrocyanide, one part $\mathrm{HCl} 2.5 \%$ and two parts of a solution containing fine iron particles of a mean diameter of 2000 nanometers (nm). This mix was dubbed (PBS Fe $24 \mathrm{~K}$ )

\section{Controls}

A human scalp hair was placed in the center of a $25 \times 75 \times 1 \mathrm{~mm}$ glass slide, two drops of the PBS $\mathrm{Fe}_{2} 4 \mathrm{~K}$ covered the hair $\mathrm{n}=10$. The preparation was allowed to evaporate for one hour. Images were recorded (Figs 1,2)

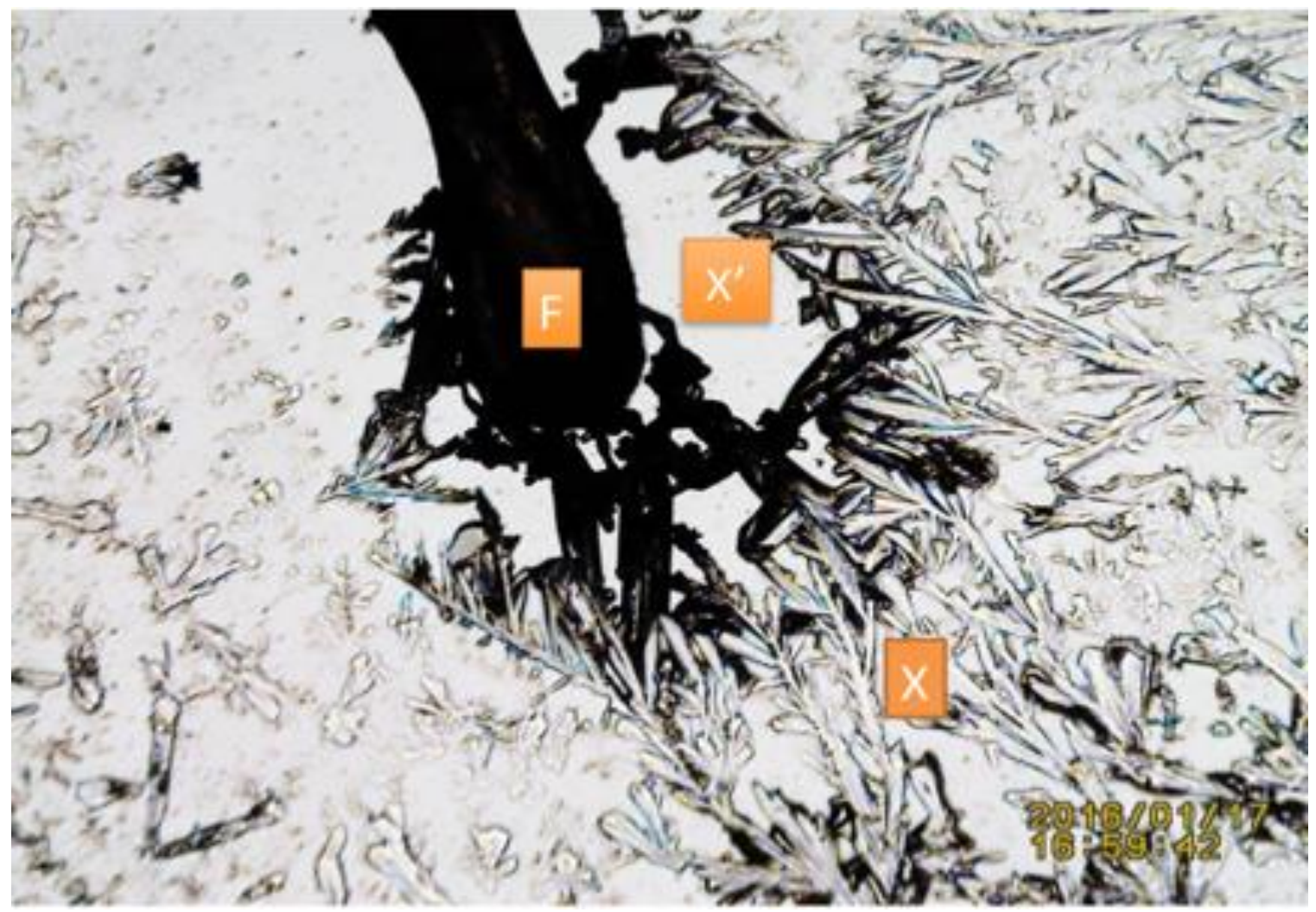

Figure 1: Control human hair in SSP PBS $\mathrm{Fe}_{2} 4 \mathrm{~K}$ after evaporation. This microphotograph depicts the arrangements of crystals of the diamagnetic Potassium Ferrocyanide surrounding the hair follicle. This image is typical of controls experiments utilizing Prussian Blue Stain mixed with fine iron particles. $\mathrm{F}=$ Follicle

$\mathrm{X}=$ Ferrocyanide crystals. $\mathrm{X}^{\prime}=$ Empty area due to diamagnetism. Please compare with Figure 4 panel C.

Image duplicated from Abraham A. Embi Bs, HUMAN TISSUE BIOMAGNETISM ATTRACTING IRON PARTICLES AS MECHANISM ELUCIDATING THE IRON AND ATHEROSCLEROSIS HYPOTHESIS 2018 International Journal of Research - Granthaalayah, (In Review) 


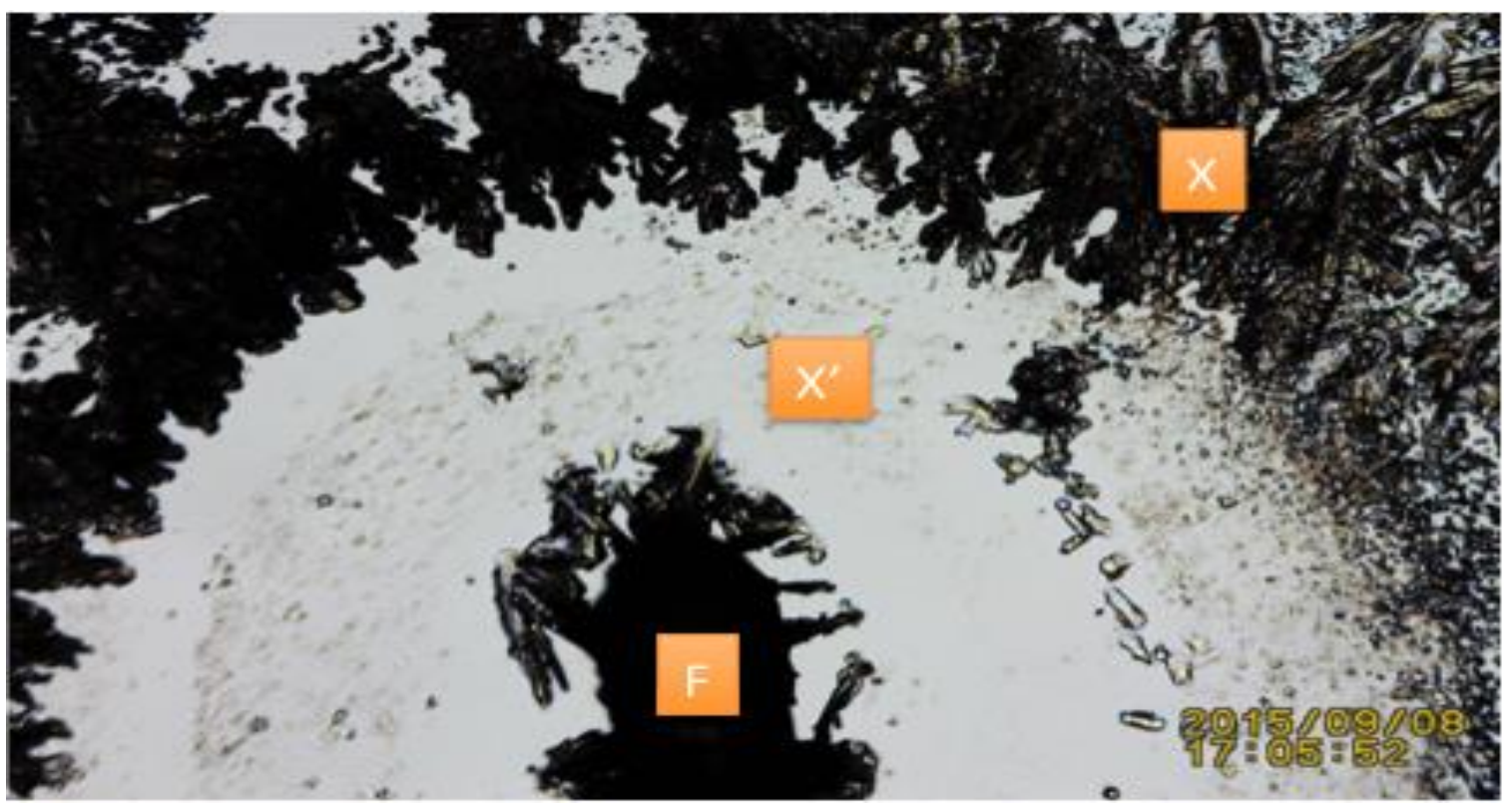

Figure 2: Another control hair on slide covered by PBS Fe2 4K solution and allowed to evaporate. Notice $\mathrm{X}=$ Diamagnetic Potassium Ferrocyanide crystals repelled by the intrinsic hair bioelectromagnetic field. $\mathrm{F}=$ Follicle. $\mathrm{X}^{\prime}=$ Empty area due to diamagnetism. Also compare with Figure 4.

\section{Ancillary Testing Blocking the CAT EMFs}

A slide assembly was developed to allow for the blocking of EMFs (emitted by CAT) by antimagnetic paint (Fig 3). One $25 \times 75 \times 1 \mathrm{~mm}$ slide was coated halfway with antimagnetic black paint (Less EMR Inc. 4 OZ can. Shielding up to 99\%). Human hairs were mounted on both sides on coverslips $(24 \times 50 \times 1 \mathrm{~mm})$ and covered by drops of PBS $\mathrm{Fe}_{2} 4 \mathrm{~K}$; then allowed to dry. Images recorded.

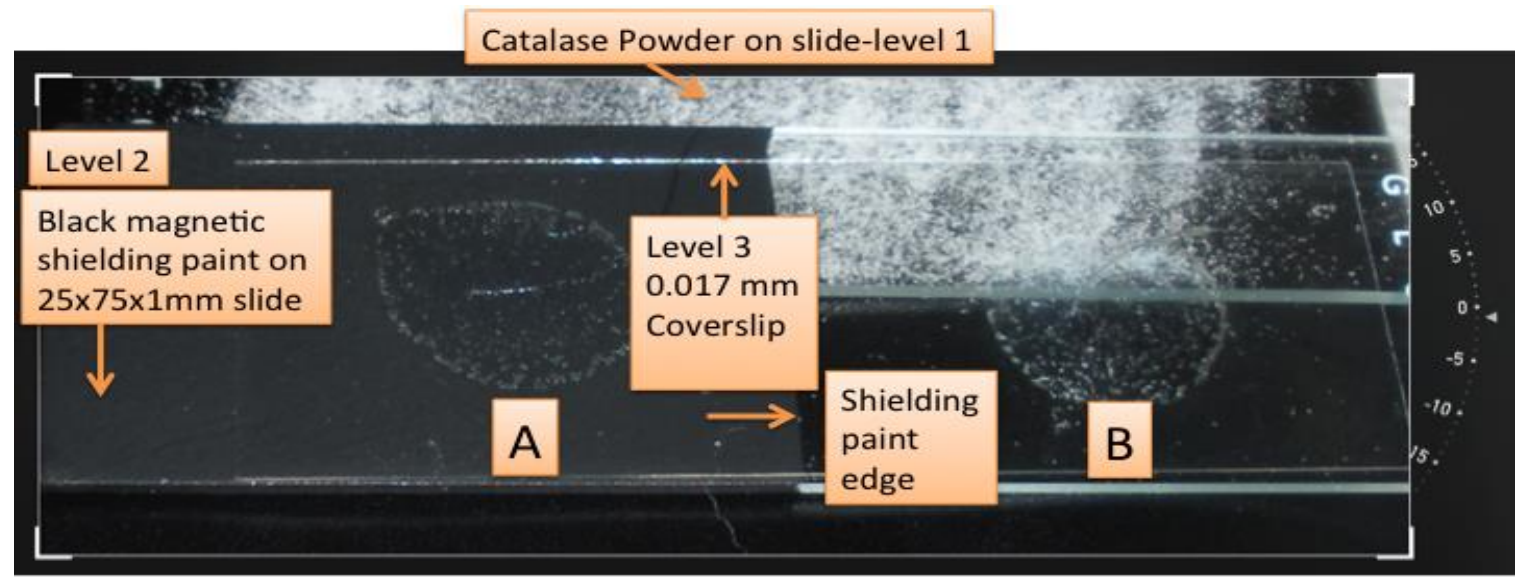

Figure 3: Photograph of slide assembly designed to magnetically shield Catalase Powder EMFs from vertically penetrating a $1 \mathrm{~mm}$ glass barrier (side A). Unshielded half of slide (side B). Slide assembly level $1=$ Smeared catalase powder, level $2=$ Glass slide with black magnetic shielding paint, level 3=0.017 mm thickness coverslip. A= Human Hair SSP PBS Fe 2 K on coverslip. This part of the coverslip is shielded by the black antimagnetic paint. $\mathrm{B}=$ Human Hair SSP PBS $\mathrm{Fe} 24 \mathrm{~K}$ on same coverslip. 
Note: Levels 2 and 3 have been manually separated (shifted downward $3 \mathrm{~mm}$ ) for demonstration purposes.

\section{Recording Equipment}

After evaporation, images were viewed and recorded in the normal mode (no filters) x10 and x40 magnifications. Equipment used was a video-microscope (Celestron LCD Digital Microscope II model \#44341, Torrance, California, USA). All pictures downloaded and labeled by using an Apple Inc. iPhoto 8.1.2, App.

\section{Results and Discussion}

The endogenous EMFs emitted during cell respiration had been proposed as an additional factor in cancer genesis (3). In previous experiments, a small fragment of processed meat were used as EMFs generator, How? By adding $\mathrm{H} 2 \mathrm{O} 2$ to the sample bubbling ensued due to the action of catalase present. This bubbling lasted approximately one hour, thus showing a significant reservoir of catalase present (2).

Since previous experiments have shown that EMFs could penetrate glass barriers (9), a slide assembly was designed to evaluate the effect of EMFs on the intact hair follicle (Fig 4). The experiment was validated by additional testing as shown in Figure 5 below.

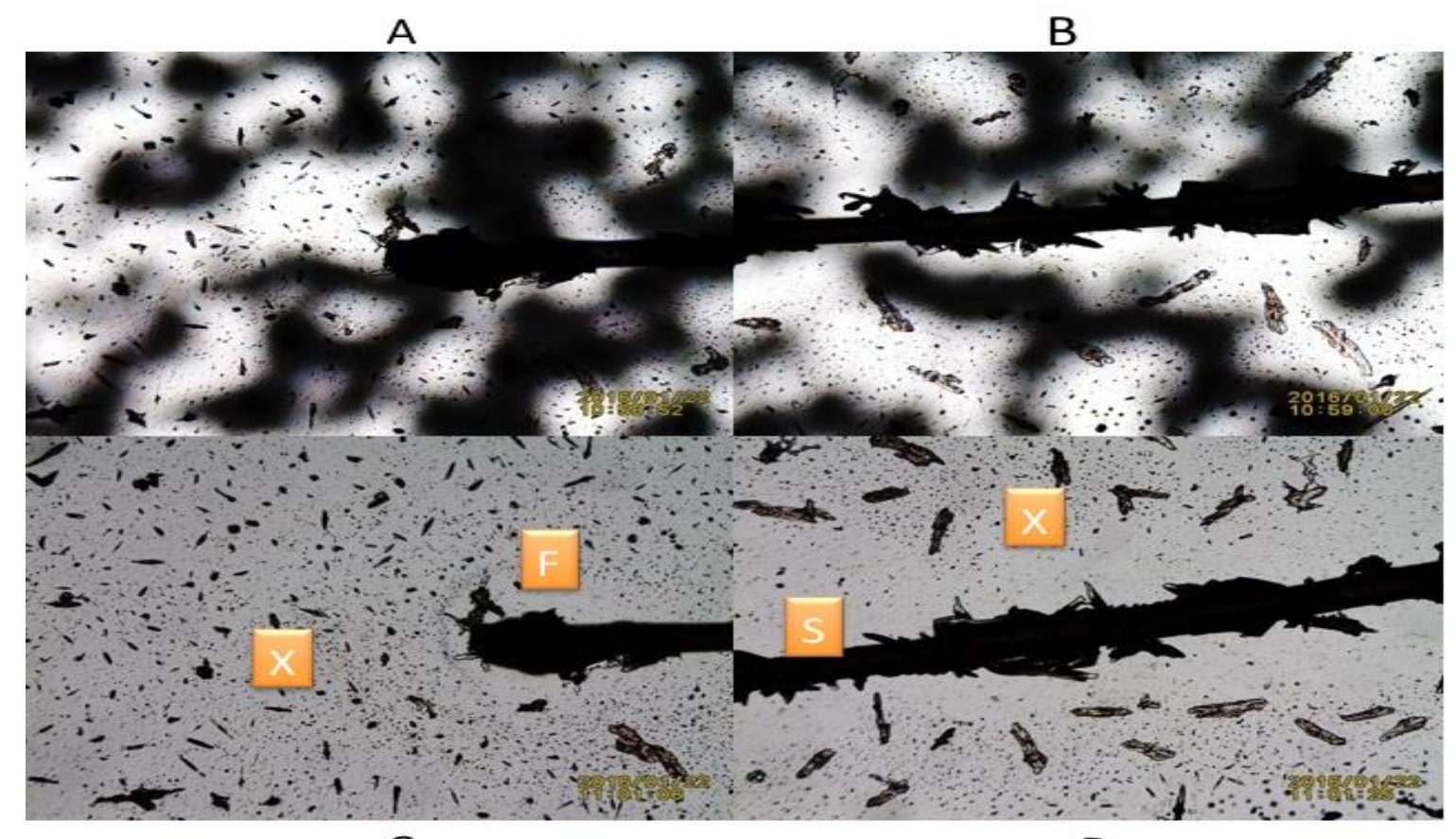

C

D

Figure 4. Panel shows a $25 \times 75 \times 1 \mathrm{~mm}$ SDW trapping catalase crystals. On top slide of SDW a human scalp hair was covered with PBS Fe2K solution and allowed to evaporate. Panels A and $\mathrm{B}$ showing the hair follicle and shaft over the catalase crystals (seen out of focus) separated by a

$1 \mathrm{~mm}$ glass barrier. In Panel $\mathrm{C}, \mathrm{X}=$ Disrupted magnetic field $\mathrm{F}=$ Hair follicle. In Panel $\mathrm{D}, \mathrm{S}=$ Hair shaft and $\mathrm{X}=$ disrupted magnetic field. The reversed side of top slide was wiped clean in order to display the hairs in detail. Please compare with control Figures $2 \& 3$. 


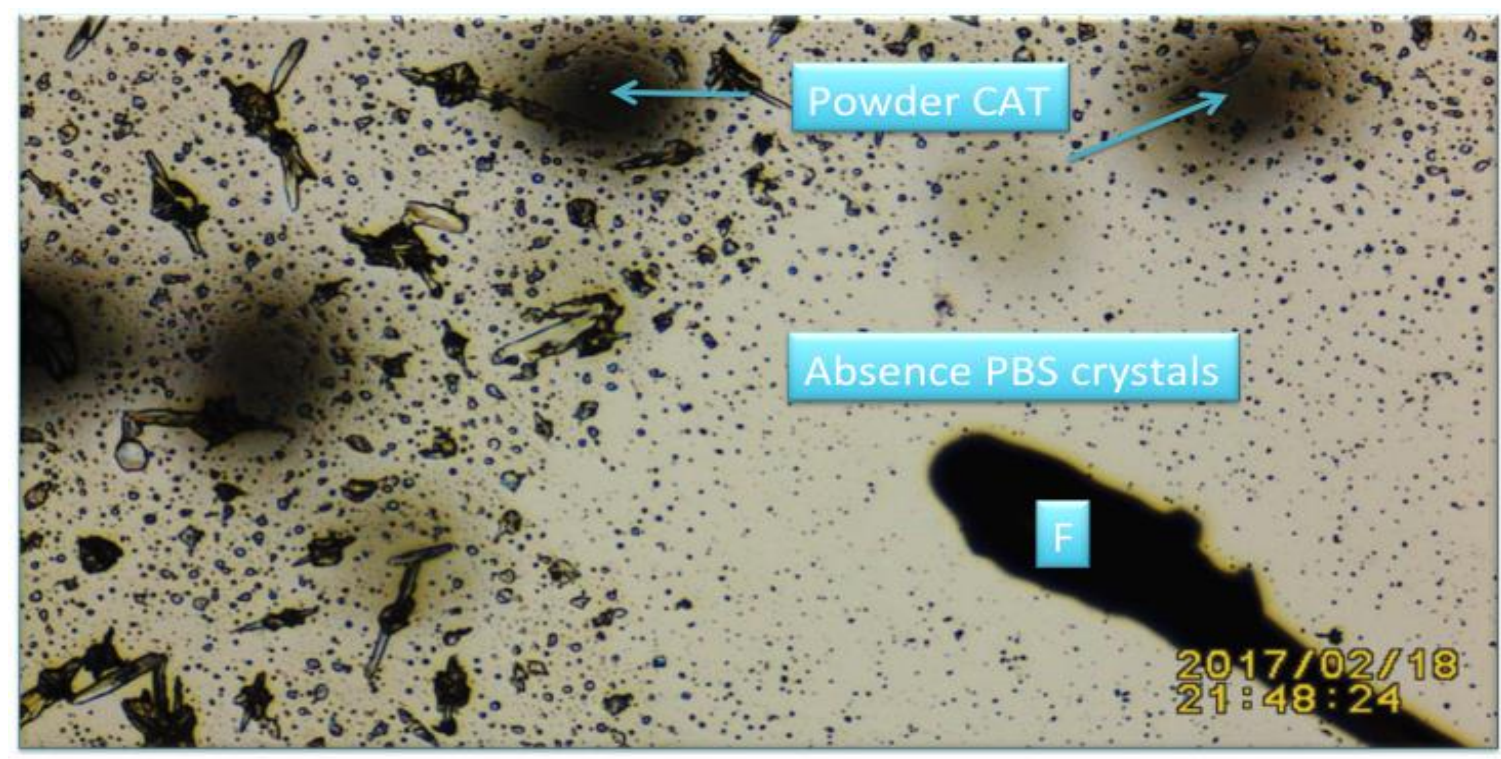

Figure 5: Human hair on SSP PBS Fe2 2K after evaporation. Catalase. Bottom layer: Powder Catalase in bottom layer on $1 \mathrm{~mm}$ glass slide SDW (out of focus). Notice the absence of large PBS crystals adhering to follicle. Compare to Panel $\mathrm{C}$ in Figure 4.

Also appreciate contrast with control Figure 6 below

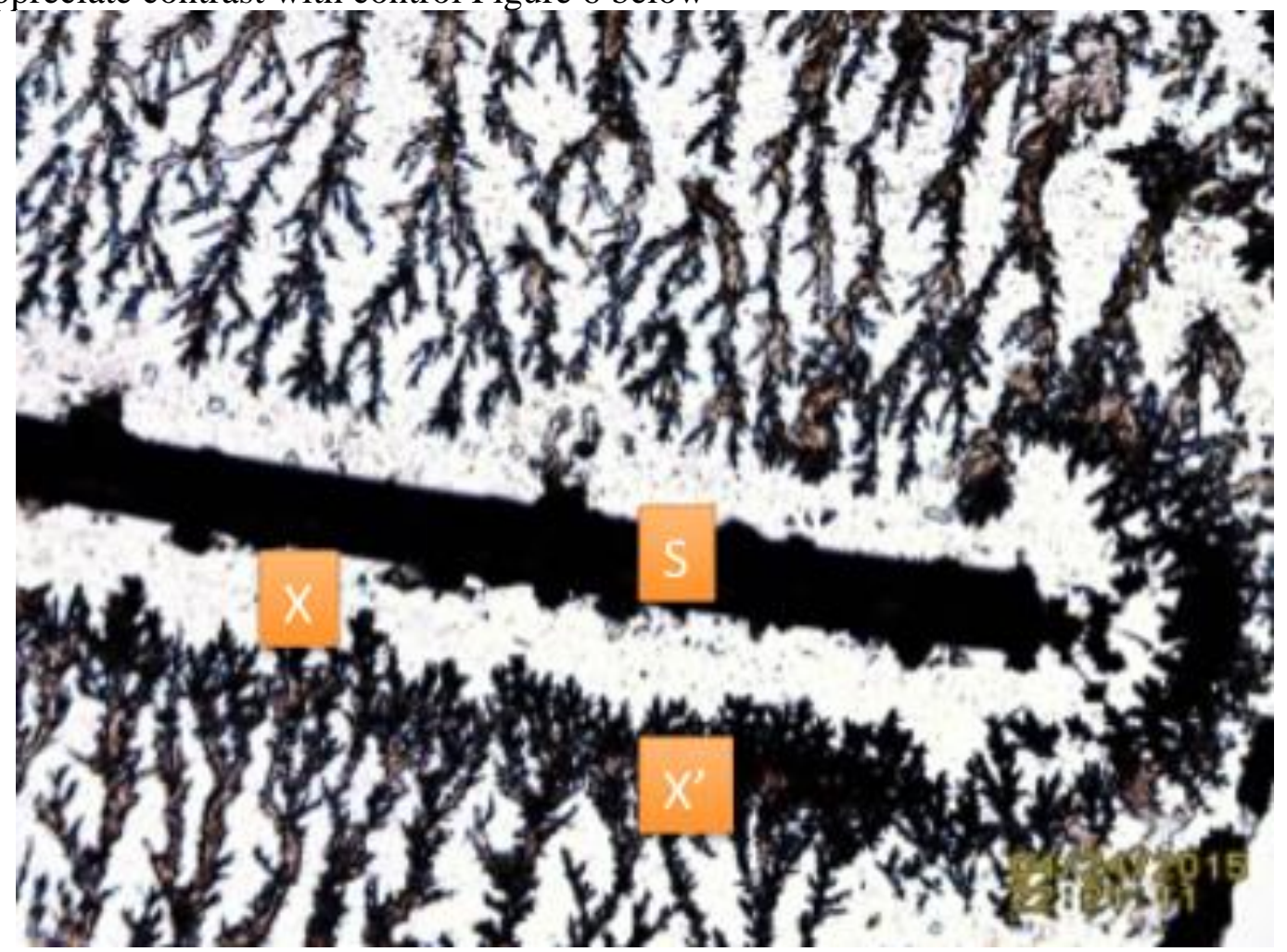

Figure 6. Control Hair. Hair shaft segment after evaporation of $\mathrm{PBS} \mathrm{Fe}_{2} 4 \mathrm{~K}$ showing

Ferrocyanide crystals (resembling a shepherd's hook) surrounding the shaft. S= Hair shaft segment $\mathrm{X}=$ Crystals separated by diamagnetic forces and $X^{\prime}=$ Iron nanoparticles mixing with Ferrocyanide crystals. 
Image reproduced from: Abraham A. Embi Bs. (2018). "THE SHEPHERDS HOOK PHENOMENON PATTERN OF HAIR ROOTS A DEMONSTRATION OF COMPARATIVE BIOLECTROMAGNETISM BETWEEN HUMAN HAIRS AND MOUSE WHISKERS BY MEANS OF THE PHOTOELECTRIC EFFECT." International Journal of Research Granthaalayah, 6(7), 317-326.

\section{Conclusions}

- The experiments have shown a change in the crystals deposition surrounding the hair follicle. This is attributed to the EMFs emitted by the enzyme catalase through a $1 \mathrm{~mm}$ glass barrier on the normal crystals accretion (read EMFs) surrounding the hair follicle. The crystals surrounding the hair are a reflection of the intrinsic hair follicle biomagnetism.

- Endogenous EMFs during cell respiration had been suggested to be cancerogenic. This by causing DNA changes.

- Exogenous catalase consumption may be a factor in cancer genesis.

- The United states Department of Agriculture (USDA) through its Food Safety and Inspection Service (FSIS) department stated when asked (Incident Case \# 151219-000012) dated December 12, 2015 as follows:

- Question: Is the level of catalase "regulated" or "inspected" by your department (either during manufacturing or as a final product) in processed meats?

- Answer: Yes, is regulated as indicated in the listing in FSIS Directive 7120.1

- Question: I have also read that catalase levels are inspected in "cooked meat" since it is always present in raw foods.

- Answer: Catalase test is conducted on cooked meat products for detecting underprocessing when the product is scheduled to be heated to the proper temperature.

In conclusion, when reading the answers, one may interpret that catalase levels are inspected in "cooked" meat products only. In "processed meat products" catalase is regulated.

- The data presented is in support of the hypothesis as stated:

The Electromagnetic Fields emitted by the protein enzyme catalase ubiquitously present in processed meat products are identified as probable factor in cancer genesis.

\section{Addendum}

Since the data introduced showed the presence of catalase in the processed meat samples tested, such as Salami, it behooves to find out whether there are governmental inspections (in my case the USA) monitoring this area:

The following questions were asked via an email dated December 22, 2015 and addressed to the United States Food Safety and Inspection Services (FSIS). Original communication on file.

Question: Is the level of catalase "regulated" or "inspected" by your department (either during manufacturing or as a final product) in processed meats? 
Answer: Yes, is regulated as indicated in the listing in FSIS Directive 7120.1

Question: I have also read that catalase levels are inspected in "cooked meat" since it is always present in raw foods.

Answer: Catalase test is conducted on cooked meat products for detecting under-processing when the product is scheduled to be heated to the proper temperature.

By reading the answers, one may interpret that catalase levels are inspected in "cooked" meat products only. In "processed meat products" catalase is regulated.

\section{References}

[1] Bouvard V, Loomis D, Guyton KZ, Grosse Y et al. Carcinogenicity of consumption of red and processed meat. International Agency for Research on Cancer Monograph Working Group. Lancet Oncol. 2015 Dec;16(16):1599-600. doi: 10.1016/S1470-2045(15)00444-1.

[2] Embi AA. Cellular respiration oxidation-reduction reactions electromagnetic fields emissions as possible causative agent in diseases: a chronic bombardment theory. Phys J. 2016;2(3):226-30.

[3] Embi AA. Endogenous electromagnetic forces emissions during cell respiration as additional factor in cancer origin. Cancer Cell International 2016 v.16; 16:60

[4] Benjamin J. Scherlag, Kaustuv Sahoo, Abraham Embi. A Novel and Simplified Method for Imaging the Electromagnetic Energy in Plant and Animal Tissue. Journal of Nanoscience and Nanoengineering 2016 Vol. 2, No. 1, 2016, pp:6-9

[5] Abraham A. Embi, Jerry I. Jacobson, Kaustuv Sahoo, Benjamin J. Scherlag. Demonstration of Inherent Electromagnetic Energy Emanating from Isolated Human Hairs.2015 JNSCI, Vol 1 No. 3 e55

[6] Schneider MR1, Schmidt-Ullrich R, Paus R. The hair follicle as a dynamic miniorgan. Curr Biol. 2009 Feb 10; 19(3): R132- 42. doi: 10.1016/j.cub.2008.12.005.

[7] Edwin Hall. On a New Action of the Magnet on Electric Currents. American Journal of Mathematics 18792 (3): 287-92

[8] Cohen D, Palti Y,Cuffin BN, Schmid SJ. Magnetic fields produced by steady currents in the body. Proc. Natl. Acad. Sci. USA 1980; 77(3): 1447-1451

[9] Embi AA, Scherlag BJ. Demonstration of Human Hair Follicle Biomagnetic Penetration Through Glass Barriers. International Journal of Materials Chemistry and Physics. 2016 Vol 2, pp. 71-74.

[10] Embi AA, Scherlag BJ. Demonstration of Biomagnetic Responses of Paired Human Hair Follicles Using Nano-size Iron Particles Solution: Inhibition of Diamagnetic Crystallization. International Journal of Materials Chemistry and Physics. 2016 Vol 2, No 2, pp:84-87

*Corresponding author.

E-mail address: embi21@ att.net 